\title{
Addendum: Independent optical excitation of distinct neural populations
}

Nathan C Klapoetke, Yasunobu Murata, Sung Soo Kim, Stefan R Pulver, Amanda Birdsey-Benson, Yong Ku Cho, Tania K Morimoto, Amy S Chuong, Eric J Carpenter, Zhijian Tian, Jun Wang, Yinlong Xie, Zhixiang Yan, Yong Zhang, Brian Y Chow, Barbara Surek, Michael Melkonian, Vivek Jayaraman, Martha Constantine-Paton, Gane Ka-Shu Wong \& Edward S Boyden Nat. Methods 11, 338-346 (2014); published online 9 February 2014; addendum published after print 28 August 2014

A trafficking variant of the Chrimson molecule (Fig. 1c) was used for the Drosophila experiments in the original version of the paper (i.e., Fig. 3, Supplementary Figs. 14-16 and Supplementary Videos 2-6). This trafficking variant, called CsChrimson-KGC-GFP-ER2, is a CsChR-Chrimson chimera in which the Chrimson $\mathrm{N}$ terminus is replaced with the CsChR N terminus (Fig. 1a and Supplementary Fig. 1), with appended KGC and ER2 trafficking sequences (Fig. 1c). In the original paper, we found $\mathrm{CsChR}$ to have high membrane expression levels (original Supplementary Figs. 5 and 6). We therefore attempted to boost Chrimson expression by swapping the Chrimson $\mathrm{N}$ terminus with the CsChR $\mathrm{N}$ terminus. As no transmembrane regions were modified, we unsurprisingly found that CsChrimson has the same spectral and kinetic properties as Chrimson in murine cultured neurons (Fig. 1b,d,f,g). We additionally compared CsChrimson with and without KGC and/or ER2 trafficking sequences and found all variants to have similar photocurrents in cultured neurons (Fig. 1d,e). However, we observed more cytosolic aggregates with the KGC version and a reduction of aggregates with the ER2 version (Supplementary Fig. 2). It is therefore likely that CsChrimson will be of use with the ER2 trafficking sequence in some biological contexts.

\section{METHODS}

Methods and any associated references are available in the online version of the paper.

Accession codes. GenBank/EMBL/DDBJ: CsChrimson is listed under accession code KJ995863.

Note: Any Supplementary Information and Source Data files are available in the online version of the paper.

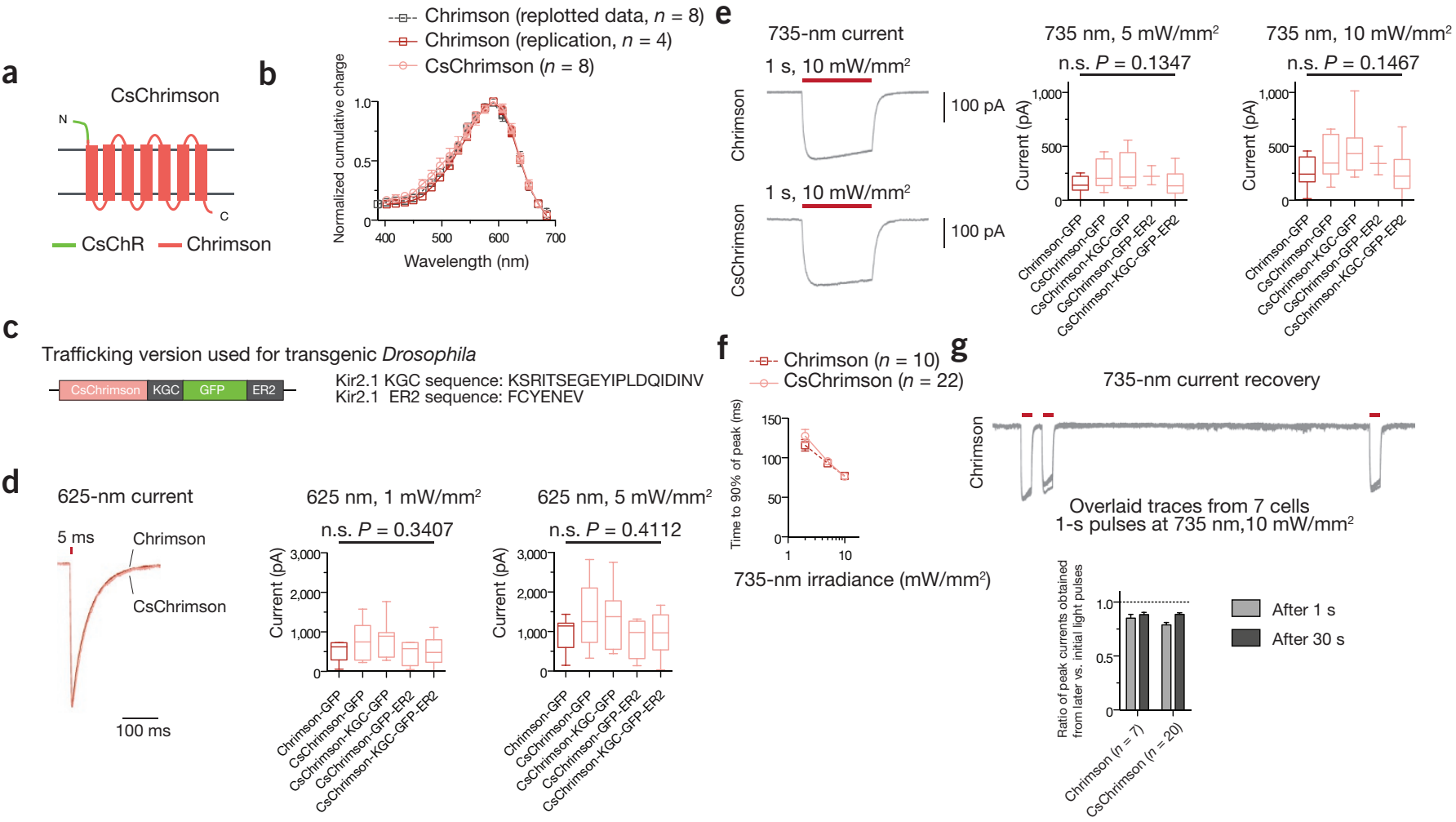

Figure 1 | CsChrimson characterization in cultured cells. (a) Schematic of the CsChrimson chimera. (b) Action spectra for Chrimson and CsChrimson as well as the Chrimson spectrum data from the original manuscript plotted in Figure 1e (HEK293 cells; measured using equal photon fluxes of $\sim 2.5 \times 10^{21}$ photons/s/ $\left.\mathrm{m}^{2}\right)$. (c) Schematic of trafficking sequences used to generate the CsChrimson Drosophila transgenics. (d,e) Maximum photocurrents in response to red (625-nm) and far-red $(735-\mathrm{nm})$ light as measured in cultured neurons. $(\mathbf{f}, \mathbf{g})$ Turn-on $(\mathbf{f})$ and recovery kinetics $(\mathbf{g})$ in response to 735 -nm light. CsChrimson kinetic data were pooled from all trafficking versions. All constructs in this panel were expressed under CaMKII promoter and selected solely on the basis of the presence of cotransfected cytosolic tdTomato expression. Illumination conditions are as labeled in each panel. Box-plot whiskers represent minimum and maximum data points. Box limits represent 25th percentile, median and 75th percentile. Chrimson-GFP: $n=9$ cells in $\mathbf{d}, n=12$ cells in e; CsChrimson-GFP: $n=7$ cells in d, $n=8$ cells in e; CsChrimson-KGC-GFP: $n=7$ cells in d,e; CsChrimson-GFP-ER2: $n=4$ cells in $\mathbf{d}, n=3$ cells in e; CsChrimson-KGC-GFP-ER2: $n=10$ cells in d, $n=11$ cells in e. Plotted data are mean \pm s.e.m. in $\mathbf{b}, \mathbf{f}, \mathbf{g}$. ANOVA with Dunnett's post hoc test with Chrimson-GFP as reference in d,e. n.s., not significant. 


\section{ONLINE METHODS}

Whole-cell patch-clamp recordings were made using a Multiclamp 700B amplifier and a Digidata 1550 digitizer (Molecular Devices). All other experimental conditions are the same as previously described. 